\title{
Interval-Valued Fuzzy Relations
}

\author{
Kur Hur, Jeong Gon Lee and Jeong Yeol Choi \\ Division of Mathematics and Informational Statistics, Nanoscale Sciences and Technology \\ Institute, Wonkwang University,Iksan, Jeonbuk, Korea 570-749
}

\begin{abstract}
By using the notion of interval-valued fuzzy relations, we forms the poset $(\operatorname{IVFR}(X), \leq)$ of interval-valued fuzzy relations on a given set $X$. In particular, we forms the subposet $(\operatorname{IVFE}(X), \leq)$ of interval-valued fuzzy equivalence relations on a given set $X$ and prove that the poset $(\operatorname{IVFE}(X), \leq)$ is a complete lattice with the least element and greatest element.
\end{abstract}

Key words : interval-valued fuzzy set [relation, equivalence relation], (complete) lattice

\section{Introduction}

After the introduction of the concept of fuzzy sets by Zadeh [11], several researchers were concerned about the generalizations of the notion of fuzzy sets, e.g., fuzzy set of type $n$ [12], intuitionistic fuzzy sets [1] and interval-valued fuzzy sets [3]. The concept of interval-valued fuzzy sets was introduced by Gorzaxczany [3], and recently there has been progress in the study of such sets by several researchers (see [2], $[4],[5],[6],[7],[8],[10])$. In [5], the topology of interval-valued fuzzy sets(IVF) is defined, and some of its properties are discussed, and then Mondal et al. [6] studied the connectedness in the topology of interval-valued fuzzy sets. Using the concept of interval-valued fuzzy sets, Jun et al. [4] introduced the notions of IVF strongly semiopen (semiclosed) sets, IVF (strong) semi-interior (IVF (strong) semiclosure), IVF strongly semiopen (semiclosed) mapping, and IVF strongly semi-continuous mapping, and then they investigated several properties. In 1992, Roy et al. [9] introduced the concept of interval-valued fuzzy relation and obtained it's fundamental results.

In this paper, by using the notion of interval-valued fuzzy relations, we forms the poset $(\operatorname{IVFR}(X), \leq)$ of interval-valued fuzzy relations on a given set $X$. In particular, we forms the subposet $(\operatorname{IVFE}(X), \leq)$ of interval-valued fuzzy equivalence relations on a given set $X$ and prove that the poset $(\operatorname{IVFE}(X), \leq)$ is a complete lattice with the least element and greatest element.

접수일자 : 2009년 1월 22일

완료일자 : 2009년 5월 19일

2000 Mathematics Subject Classification of AMS: 03E72,

03E72, 04A72. This paper was supporbed by Wonkwang

University in 2007.

\section{Preliminaries}

First we shall present the fundamental definitions given by $[3-5,7,8]$ :

Let $D(I)$ be the set of all closed subintervals of the unit interval $I$. The elements of $D(I)$ are generally denoted by capital letters $M, N, \cdots$, and note that $M=\left[M^{L}, M^{U}\right]$, where $M^{L}$ and $M^{U}$ are the lower and upper points respectively. Especially, we denote $\mathbf{0}=[0,0], \mathbf{1}=[1,1]$, and $\mathbf{a}=[a, a]$ for every $a \in(0,1)$. We also note that

$(i)(\forall M, N \in D(I))\left(M=N \Leftrightarrow M^{L}=N^{L}, M^{U}=N^{U}\right)$.

(ii) $(\forall M, N \in D(I))\left(M \leq N \Leftrightarrow M^{L} \geq N^{L}, M^{U} \leq N^{U}\right)$.

For every $M \in D(I)$, the complement of $M$, denoted by $M^{C}$, is defined by $M^{c}=1-M=\left[1-M^{U}, 1-M^{L}\right]$.

Definition 2.1 [3]. Let $X$ be a given nonempty set. A mapping $A=\left[A^{L}, A^{U}\right]: X \rightarrow D(I)$ is called an interval valued fuzzy set (briefly, IVFS) in X, where $A^{L}$ and $A^{U}$ are fuzzy sets in $X$ satisfying $A^{L}(x) \leq A^{U}(x)$ and $A(x)=\left[A^{L}(x), A^{U}(x)\right]$ for each $x \in X$.

It is clear that every fuzzy set $A$ in $X$ is an IVFS of the form $A=[A, A]$. For any $[a, b] \in D(I)$, the IVFS whose value is the interval $[a, b]$ for all $x \in X$ is denoted by $[a, b]$. In particular, for any $a \in[0,1]$, the IVFS whose value is $a=[a, a]$ for all $x \in X$ is denoted by simply $\tilde{a}$. For a point $p \in X$ and for $[a, b] \in D(I)$ with $b>0$, the IVFS which takes the value $[a, b]$ at $p$ and $\mathbf{0}$ elsewhere in $X$ is called an interval-valued fuzzy point (briefly, an IVF point) and is denoted by $[a, b]_{p}$. In particular, if $b=a$, then it is also denoted by $a_{p}$. We will denote by $D(I)^{X}$ or $\operatorname{IVF}(\mathrm{X})$ and $\operatorname{IVFp}(\mathrm{X})$ the 
set of all IVFSs and the set of all IVF points in $X$ respectively.

Notation. Let $X=\left\{x_{1}, x_{2}, \cdots, x_{n}\right\}$. Then $A=$ $\left(\left[a_{1}, b_{1}\right],\left[a_{2}, b_{2}\right], \cdots,\left[a_{n}, b_{n}\right]\right)$ denotes an IVFS in $X$ such that $A^{L}\left(x_{i}\right)=a_{i}$ and $A^{U}\left(x_{i}\right)=b_{i}$, for all $i=1,2, \cdots, n$.

Definition $2.2[3,5]$. Let $X$ be a nonempty set and let $A, B \in D(I)^{X}$. Then :

(a) $A \subset B$ iff $A^{L}(x) \leq B^{L}(x)$ and $A^{U}(x) \leq B^{U}(x)$ for all $x \in X$.

(b) $A=B$ iff $A \subset B$ and $B \subset A$.

(c) The complement $A^{c}$ of $A$ is defined by $A^{c}(x)=$ $\left[1-A^{U}(x), 1-\quad A^{L}(x)\right]$ for all $x \in X$.

(d) If $\left\{A_{i}: i \in J\right\}$ is an arbitrary subset of $D(I)^{X}$, then

$$
\begin{aligned}
& \bigcap A_{i}(x)=\left[\bigwedge_{i \in J} A_{i}^{L}(x), \bigwedge_{i \in J} A_{i}^{U}(x)\right], \\
& \bigcup A_{i}(x)=\left[\bigvee_{i \in J} A_{i}^{L}(x), \bigvee_{i \in J} A_{i}^{U}(x)\right] .
\end{aligned}
$$

Definition 2.3 [5]. Let $X$ be a nonempty set and let $A \in D(I)^{X}$. Then the set $\left\{x \in X \mid A^{U}(x)>0\right\}$ is called the support of $A$ and denoted by $\operatorname{supp}(A)$.

Definition 2.4 [5]. Let $X$ be a nonempty set and let $A \in D(I)^{X}$. Then an IVF point $M_{x}$ is said to belong to $A$, denoted by $M_{x} \tilde{\in} A$, if $A^{L}(x) \geq M^{L}$ and $A^{U}(x) \geq M^{U}$.

It is clear that $A=\bigcup\left\{M_{x}: M_{x} \tilde{\in} A\right\}$.

Result 2.A [5, Theorem 1]. Let $X$ be a nonempty set and let $A, B, C, A_{i}, B_{i} \in D(I)^{X}$. Then the following hold :

(a) $\tilde{0} \subset A \subset \tilde{1}$.

(b) $A \cup B=B \cup A, A \cap B=B \cap A$.

(c) $A \cup(B \cup C)=(A \cup B) \cup C, A \cap(B \cap C)=$ $(A \cap B) \cap C$.

(d) $A, B \subset A \cup B, A \cap B \subset A, B$.

(e) $A \cap\left(\bigcup_{i} B_{i}\right)=\bigcup_{i}\left(A \cap B_{i}\right)$.

(f) $A \cup\left(\bigcap_{i} B_{i}\right)=\bigcap_{i}\left(A \cup B_{i}\right)$.

(g) $(\tilde{0})^{c}=\tilde{1},(\tilde{1})^{c}=\tilde{0}$.

(h) $\left(A^{c}\right)^{c}=A$.

(i) $\left(\bigcup_{i} A_{i}\right)^{c}=\bigcap_{i} A_{i}^{c},\left(\bigcap_{i} A_{i}\right)^{c}=\bigcup_{i} A_{i}^{c}$.

\section{Interval-valued fuzzy relations}

Definition 3.1 [9]. Let $X$ and $Y$ be nonempty sets. Then each $R=\left[R^{L}, R^{U}\right] \in D(I)^{X \times Y}$ is called an interval-valued fuzzy relation (briefly, IVFR ) from $X$ to $Y$. For each $(x, y) \in X \times Y, R(x, y)$ estimates the interval of the strength of the link between $x$ and $y$. $\quad R^{L}(x, y)$ and $R^{U}(x, y)$ are called the maximum strength and minimum strength of the link between $x$ and $y$ respectively. In particular, each member of $D(I)^{X \times X}$ is called an interval-valued fuzzy relation in $X$.

Definition 3.2 [9].Let $R \in D(I)^{X \times Y}$. Then the inverse $R^{-1}$ of $R$ is an IVFR from $Y$ to $X$ such that $R^{-1}(y, x)=R(x, y)$ for each $(y, x) \in Y \times X$. It is clear that $R^{-1 L}(y, x)=R^{L}(x, y)$ and $R^{-1 U}(y, x)=$ $R^{U}(x, y)$.

Definition 3.3. Let $R \in D(I)^{X \times Y}$ and $S \in$ $D(I)^{Y \times Z}$. Then the composition $R \circ S$ of $S$ and $R$ is an IVFR from $X$ to $Z$ defined as follows : for each $(x, z) \in X \times Z$,

$$
\begin{array}{rl}
R & S(x, z) \\
= & {\left[\bigvee _ { y \in Y } \left(R^{L}(x, y) \wedge S^{L}(y, z),\right.\right.} \\
& \bigvee_{y \in Y}\left(R^{U}(x, y) \wedge S^{U}(y, z)\right] .
\end{array}
$$

The following is the immediate result of Definition 3.2 and 3.3 .

Proposition 3.4. Let $R_{1}, R_{2}, R_{3}, Q_{1}, Q_{2} \quad \in$ $D(I)^{X \times X}$. Then :

(a) $\left(R_{1} \circ R_{2}\right) \circ R_{3}=R_{1} \circ\left(R_{2} \circ R_{3}\right)$.

(b) If $R_{1} \subset R_{2}$ and $Q_{1} \subset Q_{2}$, then $R_{1} \circ Q_{1} \subset$ $R_{2} \circ Q_{2}$. In particular,

if $Q_{1} \subset Q_{2}$, then $R_{1} \circ Q_{1} \subset R_{2} \circ Q_{2}$.

(c) $R_{1} \circ\left(R_{2} \cup R_{3}\right)=R_{1} \circ R_{2} \cup R_{1} \circ R_{3}, R_{1} \circ\left(R_{2} \cap R_{3}\right)=$ $R_{1} \circ R_{2} \cap R_{1} \circ R_{3}$

(d) If $R_{1} \subset R_{2}$, then $R_{1}^{-1} \subset R_{2}^{-1}$.

(e) $\left(R^{-1}\right)^{-1}=R,\left(R_{1} \circ R_{2}\right)^{-1}=R_{2}^{-1} \circ R_{1}^{-1}$.

(f) $\left(R_{1} \cup R_{2}\right)^{-1}=R_{1}^{-1} \cup R_{2}^{-1},\left(R_{1} \cap R_{2}\right)^{-1}=$ $R_{1}^{-1} \cap R_{2}^{-1}$.

The following is the immediate result of proposition 3.4 (a).

Corollary 3.4. Let $R, S \in D(I)^{X \times X}$. If $R \circ S=S \circ R$, then

$$
(R \circ S) \circ(R \circ S)=(S \circ S) \circ(R \circ R)
$$

Definition 3.5 [9]. An IVFR $R$ in $X$ is called 
an interval-valued fuzzy equivalence relation (briefly, IVFER ) in $X$ if it satisfies the following conditions :

(a) it is interval-valued fuzzy reflexive, i.e., $R(x, x)=[1,1]$ for each $x \in X$,

(b) it is interval-valued fuzzy symmetric, i.e., $R^{-1}=R$,

(c) it is interval-valued fuzzy transitive, i.e., $R \circ R \subset R$.

We will denote the set of all IVFERs in $X$ as $\operatorname{IVFE}(X)$. The following is the immediate result of Definition 3.5. and proposition 3.4.

Proposition 3.6. Let $R, S \in \operatorname{IVFE}(X)$.

(a) If $R$ is interval-valued fuzzy reflexive [resp., symmetric, transitive], then so is $R^{-1}$.

(b) If $R$ is interval-valued fuzzy reflexive /resp., symmetric, transitive], then so is $R \circ R$.

(c) If $R$ is interval-valued fuzzy reflexive, then $R \subset R \circ R$.

(d) If $R$ is interval-valued fuzzy symmetric, then so are $R \cup R^{-1}, R \cap R^{-1}$ and $R \circ R^{-1}=R^{-1} \cap R$.

(e) If $R$ and $S$ are interval-valued fuzzy reflexive [resp., symmetric, transitive], then so is $R \cap S$.

(f) If $R$ and $S$ are interval-valued fuzzy symmetric, then so is $R \cup S$.

From (a), (b) and (e) of Proposition 3.6, the proofs of the following result are obvious.

Corollary 3.6-1. If $R, S \in \operatorname{IVFE}(X)$, then $R^{-1}, R$ 。 $R, R \cap S \in \operatorname{IVFE}(X)$.

The following is the immediate result of Definition 2.3 and Proposition 3.6(c).

Corollary 3.6-2. If $R \in \operatorname{IVFE}(X)$, then $R \circ R=R$.

Theorem 3.7. Let $\left\{R_{\alpha}\right\}_{\alpha \in \Gamma}$ be a nonempty family of IVFERs in $X$. Then $\bigcap_{\alpha \in \Gamma} R_{\alpha} \in \operatorname{IVFE}(X)$. However, in general, $\bigcup_{\alpha \in \Gamma} R_{\alpha}$ need not be an IVFER in $X$.

Proof. Let $x \in X$ and let $R=\bigcap_{\alpha \in \Gamma} R_{\alpha}$. Then, since each $R_{\alpha}$ is interval-valued fuzzy reflexive,

$$
R^{L}(x, x)=\bigwedge_{\alpha \in \Gamma} R_{\alpha}^{L}(x, x)=1
$$

and

$$
R^{U}(x, x)=\bigwedge_{\alpha \in \Gamma} R_{\alpha}^{U}(x, x)=1
$$

Thus $R(x, x)=[1,1]$. So $R$ is interval-valued fuzzy reflexive. It is clear that $R$ is interval-valued fuzzy symmetric. Now let $(x, z) \in X \times X$. Then

$$
\begin{aligned}
{[R \circ R]^{L} } & (x, z) \\
= & \bigvee_{y \in Y}\left[R^{L}(x, y) \wedge R^{L}(y, z)\right] \\
= & \bigvee_{y \in Y}\left[\left(\bigwedge_{\alpha \in \Gamma} R_{\alpha}^{L}(x, y)\right) \wedge\left(\bigwedge_{\alpha \in \Gamma} R_{\alpha}^{L}(y, z)\right)\right] \\
& \leq \bigwedge_{\alpha \in \Gamma}\left(\bigvee_{y \in Y}\left[R_{\alpha}^{L}(x, y) \wedge R_{\alpha}^{L}(y, z)\right]\right) \\
= & \bigwedge_{\alpha \in \Gamma}\left(R_{\alpha} \circ R_{\alpha}\right)^{L}(x, z) \\
& \leq \bigwedge_{\alpha \in \Gamma} R_{\alpha}^{L}(x, z)\left[\text { Since } R_{\alpha} \circ R_{\alpha} \subset R_{\alpha}\right] \\
= & R^{L}(x, z) .
\end{aligned}
$$

Similarly, we can see that $(R \circ R)^{U}(x, z) \leq R^{U}(x, z)$. Thus $R$ is interval-valued transitive. Hence $R=$ $\bigcap_{\alpha \in \Gamma} R_{\alpha} \in \operatorname{IVFE}(X)$.

Example 3.7. Let $X=\{a, b, c\}$ and let $R$ and $S$ be the IVFRs in $X$ represented by the following matrices, respectively :

\begin{tabular}{c|ccc}
$R$ & $a$ & $b$ & $c$ \\
\hline$a$ & {$[1,1]$} & {$[0.3,0.8]$} & {$[0.4,0.9]$} \\
$b$ & {$[0.3,0.8]$} & {$[1,1]$} & {$[0.3,0.8]$} \\
$c$ & {$[0.4,0.9]$} & {$[0.3,0.8]$} & {$[1,1]$} \\
$S$ & $a$ & $b$ & $c$ \\
\hline$a$ & {$[1,1]$} & {$[0.4,0.9]$} & {$[0.5,0.7]$} \\
$b$ & {$[0.4,0.9]$} & {$[1,1]$} & {$[0.4,0.7]$} \\
$c$ & {$[0.5,0.7]$} & {$[0.4,0.7]$} & {$[1,1]$}
\end{tabular}

Then clearly $R, S \in \operatorname{IVFE}(X)$ and $R \cup S$ is the IVFR in $X$ represented by the following matrix :

\begin{tabular}{c|ccc}
$R \cup S$ & $a$ & $b$ & $c$ \\
\hline$a$ & {$[1,1]$} & {$[0.4,0.9]$} & {$[0.5,0.9]$} \\
$b$ & {$[0.4,0.9]$} & {$[1,1]$} & {$[0.4,0.8]$} \\
$c$ & {$[0.5,0.9]$} & {$[0.4,0.8]$} & {$[1,1]$}
\end{tabular}

On the other hand,

$[(R \cup S) \circ(R \cup S)]^{U}(b, c)=0.9>0.8=(R \cup S)^{U}(b, c)$.

Thus $(R \cup S) \circ(R \cup S) \not \subset R \cup S$. So $R \cup S$ is not intervalvalued fuzzy transitive. Hence $R \cup S \notin \operatorname{IVFE}(X)$.

Proposition 3.8. Let $R$ and $S$ be interval-valued fuzzy reflexive relations in a set $X$. Then $R \circ S$ is interval-valued fuzzy reflexive. 
Proof. Let $x \in X$. Then

$$
\begin{aligned}
(R \circ S)^{L} & (x, x) \\
& =\bigvee_{y \in X}\left[S^{L}(x, y) \wedge R^{L}(y, x)\right] \\
& \geq S^{L}(x, x) \wedge R^{L}(x, x)[\text { By the hypotheses] } \\
& =1 .
\end{aligned}
$$

Similarly, we can see that $(R \circ S)^{U}(x, x) \geq 1$. Thus $(R \circ S)(x, x)=[1,1]$, for each $x \in X$. Hence this completes the proof.

Proposition 3.9. Let $R, S \in \operatorname{IVFE}(X)$. If $R \circ S=$ $S \circ R$, then $R \circ S \in \operatorname{IVFE}(X)$.

Proof. By Proposition 3.8, it is clear that $R \circ S$ is interval-valued fuzzy reflexive. Let $x, y \in X$. Then

$$
\begin{aligned}
(R \circ S)^{L}(x, y) & \\
& =\bigvee_{z \in X}\left[S^{L}(x, z) \wedge R^{L}(z, y)\right] \\
& =\bigvee_{z \in X}\left[R^{L}(y, z) \wedge S^{L}(z, x)\right]
\end{aligned}
$$

[Since $R$ and $S$ are interval-valued fuzzy symmetric] $=(S \circ R)^{L}(y, x)=(R \circ S)^{L}(y, x)$.

[Since $R \circ S=S \circ R$ ]

Similarly, we can see that $(R \circ S)^{U}(x, y)=(R \circ$ $S)^{U}(y, x)$. Thus $R \circ S$ is interval-valued fuzzy symmetric. On the other hand,

$$
\begin{aligned}
& (R \circ S) \circ(R \circ S) \\
& \quad=(R \circ R) \circ(S \circ S) \quad[\text { By Corollary 3.4] } \\
& \quad \subset R \circ S .[\text { By the hypothesis and Proposition 3.4(b)] }
\end{aligned}
$$

So $R \circ S$ is interval-valued fuzzy transitive. Hence $R \circ S \in \operatorname{IVFE}(X)$.

Let $R \in \operatorname{IVFE}(X)$ and let $a \in X$. We define a mapping $R_{a}: X \rightarrow D(I)$ as follows : for each $x \in X$,

$$
R_{a}(x)=R(a, x) .
$$

Then clearly $R_{a}$ is an IVFS in $X$. In this case, $R_{a}$ is called an interval-valued fuzzy equivalence class of $R$ containing $a$. The set $\left\{R_{a}: a \in X\right\}$ is called the interval-valued fuzzy quotient set of $X$ by $R$ and denoted by $X / R$.

Theorem 3.10. Let $R \in \operatorname{IVFE}(X)$. Then :

(a) $R_{a}=R_{b}$ if and only if $R(a, b)=[1,1]$ for any $a, b \in X$. (b) $R(a, b)=[0,0]$ if and only if $R_{a} \cap R_{b}=\mathbf{0}$ for any $a, b \in X$.

(c) $\bigcup_{a \in X} R_{a}=\mathbf{1}$.

(d) There exists the surjection $\pi: X \rightarrow X / R$ (called the natural mapping) defined by $\pi(x)=$ $R_{x}$ for each $x \in X$.

Proof. (a) $(\Rightarrow)$ : Suppose $R_{a}=R_{b}$. Since $R$ is intervalvalued fuzzy reflexive, $R(a, b)=R_{a}(b)=R_{b}(b)=$ $R(b, b)=[1,1]$. So $R(a, b)=[1,1]$.

$(\Leftarrow)$ : Suppose $R(a, b)=[1,1]$. Then $R^{U}(a, b)=1$ and $R^{L}(a, b)=1$. Let $x \in X$. Then

$$
\begin{aligned}
& R_{a}^{U}(x)=R^{U}(a, x) \\
& \quad \geq \bigvee_{z \in X}\left[R^{U}(a, z) \wedge R^{U}(z, x)\right]
\end{aligned}
$$

[Since $\mathrm{R}$ is interval-valued fuzzy transitive]

$$
\begin{aligned}
& \geq R^{U}(a, b) \wedge R^{U}(b, x)=1 \wedge R^{U}(b, x) \\
& =R^{U}(b, x)=R_{b}^{U}(x) .
\end{aligned}
$$

Similarly, $R_{a}^{L}(x) \geq R_{b}^{L}(x)$. Thus $R_{b} \subset R_{a}$. By the similar arguments, $R_{a} \subset R_{b}$. Hence $R_{a}=R_{b}$. The proofs of (b), (c) and (d) are easy. This completes the proof.

\section{The interval-valued fuzzy equivalence relation generated by an IVFR}

Definition 4.1. Let $R \in \operatorname{IVFR}(X)$ and let $\left\{R_{\alpha}\right\}_{\alpha \in \Gamma}$ be the family of all the IVFERs in $X$ containing $R$. Then $\bigcap_{\alpha \in \Gamma} R_{\alpha}$ is called the IVFER generated by $R$ and denoted by $R^{e}$.

It is clear that $R^{e}$ is the smallest IVFER containing $R$.

Definition 4.2. Let $R$ be an IVFR in $X$. Then the interval-valued fuzzy transitive closure of $R$, denoted by $\hat{R}$, is defined as follows:

$$
\hat{R}=\cup_{n \in \mathbb{N}} R^{n}, \quad \text { where } R^{n}=R \circ R \circ \cdots \circ R,
$$

in which $R$ occurs $n$ times.

The following is the immediate result of Definition 3.2 .

Proposition 4.3. Let $R$ be an IVFR in $X$. Then :

(a) $\hat{R}$ is an interval-valued fuzzy transitive relation in $X$.

(b) If there exists $n \in \mathbb{N}$ such that $R^{n+1}=R^{n}$, then $\hat{R}=R \cup R^{2} \cup \cdots \cup R^{n}$. 


\begin{tabular}{c|ccc}
$R$ & $a$ & $b$ & $c$ \\
\hline$a$ & {$[0.2,0.6]$} & {$[0,0.1]$} & {$[0.3,0.7]$} \\
$b$ & {$[0.1,0.3]$} & {$[0.1,0.5]$} & {$[0,0]$} \\
$c$ & {$[0.4,0.8]$} & {$[0,0]$} & {$[0.5,0.8]$}
\end{tabular}

Then

\begin{tabular}{c|ccc}
$R^{2}$ & $a$ & $b$ & $c$ \\
\hline$a$ & {$[0.3,0.7]$} & {$[0,0.1]$} & {$[0.3,0.7]$} \\
$b$ & {$[0,0]$} & {$[0.1,0.5]$} & {$[0.1,0.3]$} \\
$c$ & {$[0.4,0.8]$} & {$[0.4,0.8]$} & {$[0.5,0.8]$} \\
$R^{3}$ & $a$ & $b$ & $c$ \\
\hline$a$ & {$[0.3,0.7]$} & {$[0,0.1]$} & {$[0.3,0.7]$} \\
$b$ & {$[0.1,0.3]$} & {$[0.1,0.5]$} & {$[0.1,0.3]$} \\
$c$ & {$[0.4,0.8]$} & {$[0.4,0.8]$} & {$[0.5,0.8]$}
\end{tabular}

Thus $R^{2}=R^{3}$. So $\hat{R}=R \cup R^{2}$. Moreover $\hat{R} \circ \hat{R} \subset \hat{R}$.

\begin{tabular}{c|ccc}
$\hat{R}$ & $a$ & $b$ & $c$ \\
\hline$a$ & {$[0.3,0.7]$} & {$[0,0.1]$} & {$[0.3,0.7]$} \\
$b$ & {$[0.1,0.3]$} & {$[0.1,0.5]$} & {$[0.1,0.3]$} \\
$c$ & {$[0.4,0.8]$} & {$[0.4,0.8]$} & {$[0.5,0.8]$} \\
$\hat{R} \circ \hat{R}$ & $a$ & $b$ & $c$ \\
\hline$a$ & {$[0.3,0.7]$} & {$[0,0.1]$} & {$[0.3,0.7]$} \\
$b$ & {$[0.1,0.3]$} & {$[0.1,0.5]$} & {$[0.1,0.3]$} \\
$c$ & {$[0.4,0.8]$} & {$[0.4,0.8]$} & {$[0.5,0.8]$}
\end{tabular}

Hence $\hat{R}=R \cup R^{2}$ is interval-valued fuzzy transitive.

The following is the immediate result of (b) and (f) in Proposition 2.6.

Proposition 4.4. If $R$ is interval-valued fuzzy symmetric, then so is $\hat{R}$.

Proposition 4.5. Let $R$ and $S$ be IVFRs in $X$. Then

(a) If $R \subset S$, then $\hat{R} \subset \hat{S}$.

(b) If $R \circ S=S \circ R$ and $R, S \in \operatorname{IVFE}(X)$, then $(\widehat{R \circ S})=R \circ S$.

Proof. (a) It is clear from proposition 3.4 (b).

(b) Suppose $R \circ S=S \circ R$ and $R, S \in \operatorname{IVFE}(X)$. Then it is clear that $(R \circ S)^{1}=R \circ S$. Now suppose $(R \circ S)^{k}=R \circ S$ for any $k \geq 2$. Then

$$
\begin{aligned}
(R \circ S)^{k+1} & =(R \circ S)^{k} \circ(R \circ s)=(R \circ S) \circ(R \circ S) \\
& =(R \circ R) \circ(S \circ S)=R \circ S .
\end{aligned}
$$

So $(R \circ S)^{n}=R \circ S$ for any $n \geq 1$. Hence $\widehat{R \circ S}=R \circ S$

Definition 4.6 We define two mappings $\triangle, \nabla: X \rightarrow$ $D(I)$ as follows : for any $x, y \in X$,

$$
\triangle(x, y)= \begin{cases}{[1,1]} & \text { if } x=y \\ {[0,0]} & \text { if } x \neq y\end{cases}
$$

and

$$
\nabla(x, y)=[1,1]
$$

It is clear that $\triangle, \nabla \in \operatorname{IVFE}(X)$ and $R$ is an interval-valued fuzzy reflexive relation in $X$ if and only if $\triangle \subset R$.

Theorem 4.7. If $R$ is an IVFR in $X$, then $R^{e}=$ $R \cup \widehat{R^{-1}} \cup 1$.

Proof. Let $S=R \cup \widehat{R^{-1}} \cup \triangle$. Then clearly $R \subset S$. By Proposition 4.3 (a), $S$ is interval-valued fuzzy transitive. Let $x \in X$. Since $\triangle \subset S$,

$$
S^{L}(x, x) \geq \triangle^{L}(x, x)=1
$$

and

$$
S^{U}(x, x) \geq \triangle^{U}(x, x)=1 .
$$

Then $S(x, x)=[1.1]$. So $S$ is interval-valued fuzzy reflexive. It is clear that $R \cup R^{-1} \cup \triangle$ is intervalvalued fuzzy symmetric. Thus, by Proposition $4.4, S$ is interval-valued fuzzy symmetric. So $S \in \operatorname{IVFE}(X)$. Now let $K \in \operatorname{IVFE}(X)$ such that $R \subset K$. Then $\triangle \subset K$ and $R^{-1} \subset K^{-1}=K$ by Proposition 3.4(d). Thus $R \cup R^{-1} \cup \triangle \subset K$. By Proposition 3.4 (b), $\left[R \cup R^{-1} \cup \triangle\right]^{n} \subset K^{n}=K$ for any $n \geq 1$. So $S \subset K$. Hence $R^{e}=S$. This completes the proof.

Proposition 4.8. Let $R, S \in \operatorname{IVFE}(X)$. We define $R \vee S$ as follows :

$$
R \vee S=\widehat{R \cup S} .
$$

Then $R \vee S \in \operatorname{IVFE}(X)$.

Proof. By Proposition 4.3 (a), $R \vee S$ is interval-valued fuzzy transitive. Since $R$ and $S$ are interval-valued fuzzy symmetric, $R \cup S$ is interval-valued fuzzy symmetric by Proposition 3.6 (f). Thus, by Proposition 4.4, $R \vee S=\widehat{R \cup S}$ is interval-valued fuzzy symmetric. Let $x \in X$. Then

$$
\begin{aligned}
& (R \vee S)(x, x) \\
& =\left[\bigvee_{n \in \mathbb{N}}\left[R^{L}(x, x) \vee S^{L}(x, x)\right]^{n}, \bigvee_{n \in \mathbb{N}}\left[R^{U}(x, x) \vee S^{U}(x, x)\right]^{n}\right] \\
& =\left[\bigvee_{n \in \mathbb{N}}(1 \vee 1)^{n}, \bigvee_{n \in \mathbb{N}}(1 \vee 1)^{n}\right]
\end{aligned}
$$

[Since $R$ and $S$ are interval-valued fuzzy reflexive ] $=[1,1]$.

So $R \vee S$ is interval-valued fuzzy reflexive. Hence $R \vee S \in \operatorname{IVFE}(X)$.

The following result gives another description for $R \vee S$ of two IVFERs $R$ and $S$. 
Proposition 4.9. Let $R, S \in \operatorname{IVFE}(X)$. If $R$ 。 $S \in \operatorname{IVFE}(X)$, then $R \circ S=R \vee S$, where $R \vee S$ denotes the least upper bound for $\{R, S\}$ with respect to the inclusion.

Proof. Let $x, y \in X$. Then

$$
\begin{aligned}
& (R \circ S)^{L}(x, y) \\
& \quad \geq S^{L}(x, y) \wedge R^{L}(y, y) \\
& \quad=S^{L}(x, y) \wedge 1 \\
& {[\text { Since } R \text { is interval-valued fuzzy reflexive] }} \\
& \quad=S^{L}(x, y) .
\end{aligned}
$$

Similarly, we can see that $(R \circ S)^{U}(x, y) \geq$ $S^{U}(x, y)$. Thus $S \subset R \circ S$. Also, by the similar method, $R \subset R \circ S$. So $R \circ S$ is an upper bound for $\{R, S\}$ with respect to " $\subset$ ".

Now let $P \in \operatorname{IVFE}(X)$ such that $R \subset P$ and $S \subset P$. Let $x, y \in X$. Then

$$
\begin{aligned}
(R & \circ S)^{L}(x, y) \\
& =\bigvee_{z \in X}\left[S^{L}(x, z) \wedge R^{L}(z, y)\right] \\
& \leq \bigvee_{z \in X}\left[P^{L}(x, z) \wedge P^{L}(z, y)\right] \\
& =(P \circ P)^{L}(x, y) \\
& \leq P^{L}(x, y) .
\end{aligned}
$$

[ Since $P$ is interval-valued fuzzy transitive]

Similarly, we can see that $(R \circ S)^{U}(x, y) \leq$ $P^{U}(x, y)$. Thus $R \circ S \subset P$. So $R \circ S$ is the least upper bound for $\{R, S\}$ with respect to " $\subset$ ". Hence $R \circ S=R \vee S$.

Proposition 4.10. If $R, S \in \operatorname{IVFE}(X)$ such that $R \circ S=S \circ R$, then $R \vee S=\widehat{R \circ S}$.

Proof. Suppose $R, S \in \operatorname{IVFE}(X)$. Then, by Theorem 4.7,

$$
(R \cup S)^{e}=(R \cup S) \cup \widehat{(R \cup S)^{-1} \cup \triangle .}
$$

Since $R, S \in \operatorname{IVFE}(X),(R \cup S) \cup(R \cup S)^{-1} \cup \triangle=R \cup S$. By Result 2. $\mathrm{A}(\mathrm{d})$, it is clear that $R \subset R \cup S$ and $S \subset R \cup S$. Thus

$$
\begin{aligned}
R \circ S & \subset(R \cup S) \circ(R \cup S)[\text { By Proposition } 3.4(\mathrm{~b})] \\
& =R \cup S .
\end{aligned}
$$

[By Proposition 3.4 (c) and the hypothesis]
By Proposition 4.5 (a), $\widehat{R \circ S} \subset \widehat{R \cup S}$. On the other hand, since $R, S \in \operatorname{IVFE}(X)$, by Proposition 4.9, $R \subset R \circ S$ and $S \subset R \circ S$. Then $R \cup S \subset R \circ S$. Thus, by Proposition 3.5 (a), $\widehat{R \cup S} \subset \widehat{R \circ S}$. So $\widehat{R \circ S}=\widehat{R \cup S}$. Hence $R \vee S=(R \cup S)^{e}=\widehat{R \cup S}=$ $\widehat{R \circ S}$. $=\bigvee_{z \in X}\left[S^{L}(x, z) \wedge R^{L}(z, y)\right]$
tion 4.10 and Proposition $4.5(\mathrm{~b})$.

Corollary 4.10. If $R, S \in \operatorname{IVFE}(X)$ such that $R \circ S=S \circ R$, then $R \vee S=R \circ S$.

For a set $X$, it is clear that $\operatorname{IVFE}(X)$ is a poset with respect to the inclusion " $\subset$ ". Moreover, for any $R, S \in \operatorname{IVFE}(X), R \cap S$ is the greatest lower bound for $R$ and $S$ in $(\operatorname{IVFE}(X), \subset)$.

Now, we define two binary operators $\vee$ and $\wedge$ an $\operatorname{IVFE}(X)$ as follows : for any $R, S \in \operatorname{IVFE}(X)$,

$$
R \wedge S=R \cap S \quad \text { and } \quad R \vee S=(R \cup S)^{e} .
$$

Then we obtain the following result from Proposition 3.7, Definition 4.6, Proposition 4.8 and Theorem 4.10.

Theorem 4.11. (IVFE $(X), \vee, \wedge)$ is a complete lattice with the least element $\triangle$ and the greatest element $\nabla$.

\section{References}

1. K. Atanassov, "Intuitionistic fuzzy Sets", Fuzzy Sets and Systems, 20, 87-96, 1986.

2. R. Biawas, "Rosenfeld's fuzzy subgroups with interval-valued membership functions" , Fuzzy Sets and Systems, 63, 87-90, 1994.

3. M.B Gorzalzany, "A method of inference in approximate reasoning based on interval-valued fuzzy sets", Fuzzy Sets and Systems, 21, 1-17, 1987.

4. Y. B. Jun, J. J. Bae, S. H. Cho and C. S. Kim, "Interval-valued fuzzy strong semi-openness and interval-valued fuzzy strong semi-continuity", Honam Math. J., 28, no. 3, 417-431, 2006.

5. T. K. Mondal and S. K. Samanta, "Topology of interval fuzzy sets", Indian J. Pure Appl. Math., 30, no. 1, 23-38, 1999.

6. T. K. Mondal and S. K. Samanta, "Connectedness in topology of interval-valued fuzzy sets", Italian J. Pure Appl. Math., 18, 33-50, 2005.

7. J. H. Park, J. S. Park and Y. C. Kwun, "On fuzzy inclusion in the interval-valued sense", FSKG 2005, LANI 3613, Springer-Verlag, 1-10, 2005. 
8. P. V. Ramakrishnan and V. Lakshmana Gomathi Nayagam, "Hausdorff interval-valued fuzzy filters", $J$. Korean Math. Soc., 39, no. 1, 137-148, 2002.

9. M. K. Roy and R. Biswas, "I-v fuzzy relations and Sanchez's approach for medical diagnosis", Fuzzy Sets and Systems, 47, 35-38, 1992.

10. W. Zeng and Y. Shi, "Note on interval-valued fuzzy set", FSKG 2005, LANI 3613, Springer-Verlag, 20-25, 2005.

11. L. A. Zadeh, "Fuzzy sets", Inform. Control, 8, 338-353, 1965.

12. L. A. Zadeh, "The concept of a linguistic variable and its application to approximate reasoning I", Inform, Sci., 8, 199-249, 1975.

\section{저 자 소 개}

\section{Kul Hur}

He received the Ph.D degree in Department of Mathematics from Yonsei University in 1988.

From 1981 to present, he is a professor in the Division of Mathematics and informational Statistics,
Wonkwang University.

His research interest are fuzzy topology, fuzzy algebra, and category theory.

E-mail : kulhur@wonkwang.ac.kr

\section{Jeong Gon Lee}

He received the Ph.D degree in Department of Mathematics from Wonkwang University in 1998.

From 1995 to present, he is a lecturer in the Division of Mathematics and informational Statistics, Wonkwang University.

His research interest are functional analysis, fuzzy analysis, and operator theory.

E-mail : jukolee@wonkwang.ac.kr

\section{Jeong Yeol Choi}

He received the Ph.D degree in Department of Mathematics from Chonbuk University in 1984.

From 1979 to present, he is a professor in the Division of Mathematics and informational Statistics, Wonkwang University.

His research interest are functional analysis and fuzzy analysis.

E-mail : jychoi@wonkwang.ac.kr 